towards the economic integration and political unity of Africa, the Association established as a technical organization a 'Centre international d'Études et de Recherches sur l'Intégration Economique de l'Afrique' (CERINECA). Monsieur Bruno Tohngodo will serve as the first director of CERINECA. At a meeting of its scientific committee in December 1968 plans were made for organizing in 1969 a series of conferences on the theme 'Les efforts en vue des intégrations économiques en Afrique au Sud du Sahara: problèmes posés et difficultés rencontrées pour tenter de les résoudre'. These would be followed by one or more study sessions to explore this theme more fully.

The general assembly of AIDASA has issued a general appeal to university scholars, research workers, international experts, technicians, and administrators in technical co-operation to become members and to participate in the research and training projects of CERINECA. Requests for membership and further information should be addressed to the Secretary General, 14I rue des Cités, 93 Aubervilliers, France.

\title{
Vers une Nowvelle Université de Dakar
}

C'EST dans les derniers jours de mai 1968 qu'a éclaté la crise de l'Université de Dakar. Une grève générale des étudiants déclenchée par l'Union Démocratique des Étudiants Sénégalais (U.D.E.S.) et soutenue par les organisations des autres nationalités présentes sur le campus a déterminé le gouvernement à faire évacuer manu militari la cité universitaire et à fermer l'ensemble des facultés (29 mai 1968). Au cours des vacances d'été, le gouvernement et les étudiants se sont respectivement préparés à une éventuelle épreuve de force pour la rentrée d'octobre. Fort heureusement, des négociations intervinrent entre les deux parties en présence et aboutirent à un accord à la fin du mois de septembre. Sur la base de cet accord, l'Université de Dakar est partiellement rouverte pour l'année 1968/9, tandis qu'une Commission nationale de réforme prépare une refonte complète des institutions universitaires pour la rentrée d'octobre 1969 .

La crise aiguë de mai a donc abouti à un processus de reconstruction, d'adaptation et de novation, qui est actuellement en cours. Pour le moment, la Faculté de Médecine est la seule à fonctionner intégralement, tandis que certains départements seulement restent ouverts à la Faculté de Droit et des Sciences économiques, à la Faculté des Sciences, et à la Faculté des Lettres et Sciences humaines. Les principaux instituts de recherche, comme l'Institut Fondamental d'Afrique Noire et le Centre de Linguistique Appliquée de Dakar, fonctionnent normalement.

On peut admettre que la nouvelle université sera beaucoup mieux adaptée que l'ancienne aux exigences du développement au Sénégal et dans les pays voisins ou proches. Le gouvernement sénégalais souhaiterait en faire le haut lieu de l'enseignement supérieur et de la recherche fondamentale pour la zone soudano-sahélienne.

P. F.

\section{Les Nowvelles Orientations de l'I.F.A.N.}

Après le départ de $M$. Vincent Monteil, nommé Conseiller culturel auprès de l'Ambassade de France en Indonésie, c'est M. Pierre Fougeyrollas, Professeur de Psychologie sociale à la Faculté des Lettres et Sciences humaines de Dakar, qui a pris la direction de l'Institut Fondamental d'Afrique Noire.

Cet établissement demeure un institut d'université et, en tant que tel, son sort se trouve lié aux transformations en cours dans l'ensemble universitaire dakarois. Une réorganisation a été entreprise afin de subordonner toutes les activités de l'I.F.A.N. à de stricts critères d'efficacité et de rendement, qui sont indispensables pour lier la recherche fondamentale à la grande tâche de la liquidation du sous-développement africain.

Entre la recherche fondamentale, qui demeure son premier souci, et les recherches 